\title{
A Mathematical Study on a Diseased Prey-Predator Model with Predator Harvesting
}

\author{
Srinivasarao Thota \\ Department of Applied Mathematics \\ School of Applied Natural Sciences \\ Adama Science and Technology University \\ Post Box No. 1888, Adama, Ethiopia \\ Email: srinivasarao.thota [AT] astu.edu.et
}

\begin{abstract}
In this paper, we present a mathematical model for a prey-predator system with infectious disease in the prey population. We assumed that there is harvesting from the predator and a defensive property against predation. This model is constituted by a system of nonlinear decoupled ordinary first order differential equations, which describe the interaction among the healthy prey, infected prey and predator. The existence, uniqueness and boundedness of the system solutions are investigated. Local stability of the system at equilibrium points is discussed.
\end{abstract}

Keywords--- Pre-predator model, Equilibrium points, Stability Analysis, Non-linear differential equations

\section{INTRODUCTION}

The prey-predator species interaction has been studied by many researchers and engineers since Lotka-Volterra model see, for example, [1]. Similarly, the susceptible-infected-recovered population interaction is becoming an interesting research work since the pioneering work of Kermack and Mc Kendrick [2]. The dynamics of disease with in the ecological systems is playing an important role in eco-epidemiology research. In [3], Anderson and May were introduced the research on combination of these two systems, and the term "eco-epidemiology" is used first by Chattopadhyay and Arino in [4] for such type of models. Several scientists and engineers are studied the dynamics of prey-predator using various effects of variety of biological factors within the last decades, see, for example [5-8], and different types of mathematical models have been created in epidemiology using different types of incidence rates and disease, see, for example [9-13]. In this paper, we propose a mathematical model for a prey-predator system with infectious disease in the prey population. We assumed that there is harvesting from the predator and a defensive property against predation. The existence, uniqueness and boundedness of the system solutions are investigated. Local stability of the system at equilibrium points is discussed and the analytical results obtained in proposed model are justified using numerical simulations.

The paper is organized as follows. Section 2 presents the mathematical model formulation; Section 3 discusses the local stability of all possible equilibrium points of the system; and in Section 4, we discuss the conclusion of the proposed model.

\section{MODEL FORMULATION}

In the proposed model, we study a prey-predator system involving infected disease in prey. We assumed that there is harvesting from the predator and a defensive property against predation. In this model, the population density of prey is divided into two parts namely, the susceptible population density at time $t$ given by $x(t)$ and the infected population density at time $t$ denoted by $y(t)$, and the population density of predator at time $t$ is given by $z(t)$.

\subsection{Assumptions}

According to the following hypotheses, the mathematical model of an eco-epidemic prey-predator model with harvesting in predator is formulated.

(a). The prey population grows logistically with intrinsic growth rate $r>0$. It is assumed that the infected can't reproduce rather than that it competes with the susceptible individuals for food and space.

(b). The susceptible prey population becomes infected by contact with the infected prey according to the simple mass action kinetics with $\beta>0$ as the rate of infection. 
(c). The constants $e_{1} \in(0,1)$ and $e_{2} \in(0,1)$ are the conversion rates from susceptible and infected preys to predator respectively.

(d). The disease cases a death in the infected population that represented by diseased death rate $d_{1}>0$. While in the absence of prey, the predator decay exponentially with natural death rate $d_{2}>0$.

(e). We use the coefficients $\alpha>0$ and $\gamma>0$ for the competing coefficient of $y$ over $x$ and the computing coefficient of $z$ over $y$ respectively.

\subsection{Mathematical Model}

According to the above set of hypotheses the dynamics of a diseased prey-predator model with predator harvesting can be describe in the following set of first order nonlinear differential equations.

$$
\left.\begin{array}{l}
\frac{d x}{d t}=r x-\frac{\beta}{1+y} x y-\alpha x z \\
\frac{d y}{d t}=-d_{1} y+\frac{\beta}{1+y} x y-\gamma y z \\
\frac{d z}{d t}=-d_{2} z+e_{1} \alpha x z+e_{2} \gamma y z-e z
\end{array}\right\}
$$

where $x(0) \geq 0, y(0) \geq 0, z(0) \geq 0$.

\section{STABILITY ANALYSIS}

In this section, we discuss about the equilibrium points of the model (1), existence and stability analysis of equilibrium points.

\subsection{Equilibrium Points}

The equilibrium points of the system are necessary for the purpose of studying the local stability nature of the eco-epidemic prey-predator model. The system (1), under investigation, has the following four equilibrium points.

(i). Fully washed state or extent state: $E_{1}=(0,0,0)$

(ii). Infected species washed state: $E_{2}=\left(\frac{d_{2}+e}{\alpha e_{1}}, 0, \frac{r}{\alpha}\right)$

(iii). Predator washed state: $E_{3}=\left(\frac{d_{1}}{\beta-r}, \frac{r}{\beta-r}, 0\right)$. This equilibrium point exists when $\beta>r$.

(iv). Coexistence state: $E_{4}=\left(x^{*}, y^{*}, z^{*}\right)$, where

$$
\begin{aligned}
& x^{*}=\frac{\left(e_{2} \gamma+d_{2}+e\right)\left(\alpha d_{1}+\gamma r\right)-\left(d_{2}+e\right) \beta \gamma}{\alpha\left[\left(\alpha d_{1}+\gamma r\right) e+\beta \gamma\left(e_{2}-e_{1}\right)\right]}, y^{*}=\frac{\beta\left(d_{2}+e\right)-\left(\alpha d_{1}+\gamma r\right)}{\alpha\left[\left(\alpha d_{1}+\gamma r\right) e+\beta \gamma\left(e_{2}-e_{1}\right)\right]} \text { and } \\
& z^{*}=\frac{\alpha d_{1} e_{1}+e_{2} \gamma r+\left(d_{2}+e\right)(r-\beta)}{\alpha\left[\left(d_{2}+e\right)+\gamma\left(e_{2}-e_{1}\right)\right]} .
\end{aligned}
$$

This equilibrium point $E_{4}$ exists when $e_{2}>e_{1}, \quad\left(e_{2} \gamma+d_{2}+e\right)\left(\alpha d_{1}+\gamma r\right)>\left(d_{2}+e\right) \beta \gamma \quad$ and $\beta\left(d_{2}+e\right)>\left(\alpha d_{1}+\gamma r\right)$.

\subsection{Existence and Stability Analysis of Equilibrium Points}

The Jacobin matrix for the system (1) at equilibrium point $E=(x, y, z)$ is given by 


$$
J_{E}=\left(\begin{array}{ccc}
r-\frac{\beta y}{1+y}-\alpha z & -\frac{\beta x}{1+y}+\frac{\beta x y}{(1+y)^{2}} & -\alpha x \\
\frac{\beta y}{1+y} & -d_{1}+\frac{\beta x}{1+y}-\frac{\beta x y}{(1+y)^{2}}-\gamma z & -\gamma y \\
\alpha e_{1} z & e_{2} \gamma z & \alpha e_{1} x+e_{2} \gamma y-d_{2}-e
\end{array}\right)
$$

Based on the nature of Eigen values, the dynamical system (1) gets stable when all three Eigen values are negative in case of real roots or negative real parts in case of complex roots of the characteristic equation for the above Jacobin matrix (2), otherwise the dynamical system is unstable.

Theorem 1: The dynamical system (1) is unstable at the equilibrium points $E_{1}$ and $E_{2}$.

Proof: (i) The Eigen values of the dynamical system (1) at $E_{l}=(0,0,0)$ are computed using the Jacobin matrix at $E_{l}$.

$$
J_{E_{1}}=\left(\begin{array}{ccc}
r & 0 & 0 \\
0 & -d_{1} & 0 \\
0 & 0 & -d_{2}-e
\end{array}\right)
$$

Now the Eigen values are $r,-d_{1}$ and $-d_{2}-e$. Therefore, the equilibrium point $E_{l}$ is saddle point.

(ii) Similarly, the Eigen values of the dynamical system (1) at $E_{2}=\left(\frac{d_{2}+e}{\alpha e_{1}}, 0, \frac{r}{\alpha}\right)$ are computed using the Jacobin matrix at $E_{2}$.

$$
J_{E_{2}}=\left(\begin{array}{ccc}
0 & -\frac{\beta\left(d_{2}+e\right)}{\alpha e_{1}} & -\frac{d_{2}+e}{e_{1}} \\
0 & -\frac{\alpha d_{1} e_{1}+e_{1} \gamma r-\beta d_{2}-\beta e}{\alpha e_{1}} & 0 \\
r e_{1} & \frac{r e_{2} \gamma}{\alpha} & 0
\end{array}\right) .
$$

The characteristic equation of $J_{E_{2}}$ is

$$
\lambda^{3}+\left[\frac{\alpha d_{1} e_{1}+\gamma r e_{2}-\beta\left(d_{2}+e\right)}{\alpha e_{1}}\right] \lambda^{2}+\left[r\left(d_{2}+e\right)\right] \lambda+\left[\frac{r\left(d_{2}+e\right)\left(\alpha d_{1}+\gamma r\right) e_{1}-\beta\left(d_{2}+e\right)}{\alpha e_{1}}\right]=0,
$$

and the Eigen values are $\pm i \sqrt{\left(d_{2}+e\right) r}$ and $\frac{\beta\left(d_{2}+e\right)-\left(\alpha d_{1}+\gamma r\right) e_{1}}{\alpha e_{1}}$. Since there is no real part exists in case of complex Eigen values, the dynamical system is unstable.

Theorem 2: The dynamical system (1) is locally asymptotically stable at $E_{3}=\left(\frac{d_{1}}{\beta-r}, \frac{r}{\beta-r}, 0\right)$ if $r>\beta$ and $\beta^{2}>r\left(\beta+1 / 4 d_{1}\right), \beta>r$.

Proof: For this equilibrium point the corresponding Jacobin matrix is 


$$
J_{E_{3}}=\left(\begin{array}{ccc}
0 & -\frac{d_{1}(\beta-r)}{\beta} & -\frac{\alpha d_{1}}{\beta-r} \\
r & -\frac{r d_{1}}{\beta} & -\frac{\gamma r}{\beta-r} \\
0 & 0 & \frac{\alpha d_{1} e_{1}+\gamma r e_{2}+\left(d_{2}+e\right)(r-\beta)}{\beta-r}
\end{array}\right)
$$

The corresponding Eigen values are $-\frac{d_{1} r}{2 \beta} \pm i \frac{\sqrt{\left(d_{1} r\right)\left[\beta^{2}-r\left(\beta+1 / 4 d_{1}\right)\right]}}{\beta},-\frac{\left[\left(\alpha d_{1} e_{1}+\gamma r e_{2}\right)+(r-\beta)\left(d_{2}+e\right)\right]}{r-\beta}$.

Hence the system (1) is locally asymptotically stable when $r>\beta$ and $\beta^{2}>r\left(\beta+1 / 4 d_{1}\right), \beta>r$.

Theorem 3: The equilibrium point $E_{4}=\left(x^{*}, y^{*}, z^{*}\right)$ exists if $e_{2}>e_{1}, \quad \beta\left(d_{2}+e\right)>\left(\alpha d_{1}+\gamma r\right)$ and $\left(e_{2} \gamma+d_{2}+e\right)\left(\alpha d_{1}+\gamma r\right)>\left(d_{2}+e\right) \beta \gamma$.

Proof: Let $x^{*}, y^{*}, z^{*}$ be the positive solutions of the equations

$$
\begin{aligned}
& \frac{d x^{*}}{d t}=r x^{*}-\frac{\beta}{1+y^{*}} x^{*} y^{*}-\alpha x^{*} z^{*} \\
& \frac{d y^{*}}{d t}=-d_{1} y^{*}+\frac{\beta}{1+y^{*}} x^{*} y^{*}-\gamma y^{*} z^{*} \\
& \frac{d z^{*}}{d t}=-d_{2} z^{*}+e_{1} \alpha x^{*} z^{*}+e_{2} \gamma y^{*} z^{*}-e z^{*}
\end{aligned}
$$

Solving above equations for $x^{*}, y^{*}, z^{*}$, we obtain

$$
\begin{aligned}
& x^{*}=\frac{\left(e_{2} \gamma+d_{2}+e\right)\left(\alpha d_{1}+\gamma r\right)-\left(d_{2}+e\right) \beta \gamma}{\alpha\left[\left(\alpha d_{1}+\gamma r\right) e+\beta \gamma\left(e_{2}-e_{1}\right)\right]}, y^{*}=\frac{\beta\left(d_{2}+e\right)-\left(\alpha d_{1}+\gamma r\right)}{\alpha\left[\left(\alpha d_{1}+\gamma r\right) e+\beta \gamma\left(e_{2}-e_{1}\right)\right]} \text { and } \\
& z^{*}=\frac{\alpha d_{1} e_{1}+e_{2} \gamma r+\left(d_{2}+e\right)(r-\beta)}{\alpha\left[\left(d_{2}+e\right)+\gamma\left(e_{2}-e_{1}\right)\right]} .
\end{aligned}
$$

These would be positive when $e_{2}>e_{1},\left(e_{2} \gamma+d_{2}+e\right)\left(\alpha d_{1}+\gamma r\right)>\left(d_{2}+e\right) \beta \gamma$ and $\beta\left(d_{2}+e\right)>\left(\alpha d_{1}+\gamma r\right)$. So, the interior equilibrium point $E_{4}$ for system (1) exists if $e_{2}>e_{1},\left(e_{2} \gamma+d_{2}+e\right)\left(\alpha d_{1}+\gamma r\right)>\left(d_{2}+e\right) \beta \gamma$ and $\beta\left(d_{2}+e\right)>\left(\alpha d_{1}+\gamma r\right)$.

Theorem 4: The dynamical system (1) at the coexistent equilibrium point $E_{4}$ is locally asymptotically stable if $a_{0}>0, a_{2}>0$ and $a_{0} a_{1}-a_{2}>0$ otherwise is unstable.

Proof: The Jacobin matrix corresponding to the equilibrium point $E_{4}$ is

$$
J_{E_{4}}=\left(\begin{array}{ccc}
0 & H_{12} & H_{13} \\
H_{21} & H_{22} & H_{23} \\
H_{31} & H_{32} & 0
\end{array}\right)
$$

where 


$$
\begin{aligned}
H_{12}= & -\frac{\left(e_{2} \gamma\left(\alpha d_{1}+\gamma r\right)+\left(d_{2}+e\right)\left(\alpha d_{2}+\gamma(r-\beta)\right)\left(e_{1}\left(\alpha d_{1}-\beta \gamma\right)+\left(\beta e_{2}+r e_{1}\right) \gamma\right)\right.}{\alpha \beta\left(\left(d_{2}+e\right)+\gamma\left(e_{2}-e_{1}\right)\right)^{2}}, \\
H_{13}= & -\frac{\left(e_{2} \gamma\left(\alpha d_{1}+\gamma r\right)+\left(d_{2}+e\right)\left(\alpha d_{2}+\gamma(r-\beta)\right)\right.}{\left(e_{1}\left(\alpha d_{1}-\beta \gamma\right)+\left(\beta e_{2}+r e_{1}\right) \gamma\right)}, \\
H_{21}= & -\frac{\left(\left(\alpha d_{1}+\gamma r\right) e_{1}-\beta\left(d_{2}+e\right)\right)}{\left(\left(d_{2}+e\right)+\gamma\left(e_{2}-e_{1}\right)\right)}, \\
H_{22}= & \frac{1}{\alpha \beta\left(\left(d_{2}+e\right)+\gamma\left(e_{2}-e_{1}\right)\right)}\left(e_{1} e_{2} \gamma\left(\alpha d_{1}+\gamma r\right)^{2}+\left(\alpha d_{1} d_{2} e_{1}+\alpha d_{1} e_{1} e\right)\left(\alpha d_{1}-\beta \gamma\right)\right. \\
& +\left(d_{2}+e\right)\left(2 \alpha d_{1} e_{1} \gamma r-\alpha \beta d_{1} e_{2} \gamma+e_{1} \gamma^{2} r^{2}\right)-\left(e_{1}+e_{2}\right)\left(\beta d_{2} \gamma^{2} r+\beta e \gamma^{2} r\right) \\
& \left.+\left(d_{2}+2 e\right)\left(-\alpha \beta d_{1} d_{2}+\beta^{2} d_{2} \gamma-\beta d_{2} \gamma r\right)-\alpha \beta d_{1} e^{2}+\beta^{2} \gamma e^{2}-\beta e^{2} \gamma r\right), \\
H_{23}= & \frac{\left(\gamma\left(e_{1}\left(\alpha d_{1}+\gamma r\right)-\beta\left(d_{2}+e\right)\right)\right.}{\left(e_{1}\left(\alpha d_{1}-\beta \gamma\right)+\left(\beta e_{2}+r e_{1}\right) \gamma\right)}, \\
H_{31}= & \frac{\left(\alpha d_{1} e_{1}+e_{2} \gamma r+\left(d_{2}+e\right)(r-\beta)\right) e_{1}}{\left(\left(d_{2}+e\right)+\gamma\left(e_{2}-e_{1}\right)\right)}, \\
H_{32}= & \frac{\left(\alpha d_{1} e_{1}+e_{2} \gamma r+\left(d_{2}+e\right)(r-\beta)\right) e_{2} \gamma}{\left(\left(d_{2}+e\right)+\gamma\left(e_{2}-e_{1}\right)\right) \alpha}
\end{aligned}
$$

The characteristic equation $J_{E_{4}}$ is $\lambda^{3}+a_{0} \lambda^{2}+a_{1} \lambda+a_{2}=0$. Here $a_{0}=-H_{22}$, $a_{1}=-\left(H_{12} H_{21}+H_{13} H_{31}+H_{23} H_{32}\right)$ and $a_{2}=-H_{31} H_{12} H_{23}-H_{13} H_{21} H_{32}+H_{13} H_{22} H_{31}$. Therefore, by RouthHurwitz criteria, the equilibrium point $E_{4}$ is locally asymptotically stable if $a_{0}>0, a_{2}>0$ and $a_{0} a_{1}-a_{2}>0$ otherwise is unstable.

\section{CONCLUSIONS AND DISCUSSIONS}

This paper elucidates an ecological model of a prey-predator system with infectious disease in the prey population. This model is constituted by a system of nonlinear decoupled ordinary first order differential equations, which describe the interaction among the healthy prey, infected prey and predator. By using perturbed method, we identify the local stability nature of the system at each possible equilibrium point and also the existence, uniqueness and boundedness of the system solutions are investigated.

(i). From Theorem 2, we can observe that the dynamical system (1) is locally asymptotically stable at the equilibrium point $E_{3}$ when $r>\beta$ and $\beta^{2}>r\left(\beta+1 / 4 d_{1}\right), \beta>r$.

(ii). One can also notice, from Theorem 4 , that the dynamical system (1) at the coexistent equilibrium point $E_{4}=($ $\left.x^{*}, y^{*}, z^{*}\right)$ is locally asymptotically stable if $a_{0}>0, a_{2}>0$ and $a_{0} a_{1}-a_{2}>0$ otherwise is unstable, where $a_{1}=-\left(H_{12} H_{21}+H_{13} H_{31}+H_{23} H_{32}\right), a_{2}=-H_{31} H_{12} H_{23}-H_{13} H_{21} H_{32}+H_{13} H_{22} H_{31}, a_{0}=-H_{22}$, and $H_{i j}, 1 \leq i, j \leq 3$ are as given Theorem 4 .

\section{REFERENCES}

[1]. M. R. May, Stability and Complexity in Model Ecosystems. Princeton, NJ: Princeton University, 1973.

[2]. Kermack WO, Mc Kendrick AG. Contributions to the mathematical theory of epidemics, part1. Proceedings of the Royal Society of London Series A1927; 115:700-721.

[3]. Anderson RM, May RM. The invasion, persistence and spread of infectious diseases within animal and plant communities. Philosophical Transactions of the Royal Society of London. Series B 1986; 314:533-570.

[4]. Chattopadhyay J, Arino O. A predator-prey model with disease in the prey. Nonlinear Analysis 1999; 36:747-766. 
[5]. Z. Ma, F. Chen, C. Wu, and W. Chen, "Dynamic behaviors of a Lotka-Volterra predator-prey model incorporating a prey refuge and predator mutual interference," Applied Mathematics and Computation, vol. 219, no. 15, pp. 79457953, 2013.

[6]. P. J. Pal and P. K. Mandal, "Bifurcation analysis of a modified Leslie-Gower predator-prey model with BeddingtonDeAngelis functional response and strong Allee effect," Mathematics and Computers in Simulation, vol. 97, pp. 123-146, 2014.

[7]. Raid Kamel Naji and Salam Jasim Majeed. The Dynamical Analysis of a Prey-Predator Model with a Refuge-Stage Structure Prey Population. International Journal of Differential Equations. Volume 2016, Article ID 2010464, 10 pages.

[8]. Ahmed Sami Abdulghafour and Raid Kamel Naji. The impact of refuge and harvesting on the dynamics of preypredator system. Sci. Int. (Lahore), 30 (2), 315-323. 2018

[9]. D. Greenhalgh, Q. J. A. Khan, and F. I. Lewis, "Hopf bifurcation in two SIRS density dependent epidemic models," Mathematical and Computer Modelling, vol. 39, no. 11-12, pp. 1261-1283, 2004.

[10]. T. L. Zhang, J. L. Liu, and Z. D. Teng, "Stability of Hopf bifurcation of a delayed SIRS epidemic model with stage structure," Nonlinear Analysis: Real World Applications, vol. 11, no. 1, pp. 293-306, 2010.

[11]. Y. Z. Pei, S. Li, C. Li, and S. Chen, "The effect of constant and pulse vaccination on an SIR epidemic model with infectious period," Applied Mathematical Modelling, vol. 35, no. 8, pp. 3866-3878, 2011.

[12]. Raid Kamel Naji and Burhan Haqi Abdulateef. The dynamics of SICIR model with nonlinear incidence rate and saturated treatment function. Sci. Int. (Lahore), 29 (6), 1223-1236, 2017.

[13]. S. Thota: Prey-Predator Model for Awash National Park, Oromia, Ethiopia and Its Stability Analysis with Simulations, Journal of Science and Sustainable Development, 7 (2) (2019), 15-21. 